An operator for graph parameters between $\alpha$ and $\bar{\chi}$

\title{
The sandwich line graph
}

\author{
Denis Cornaz
}

Université Blaise Pascal-Clermont-Ferrand II 
Introduction

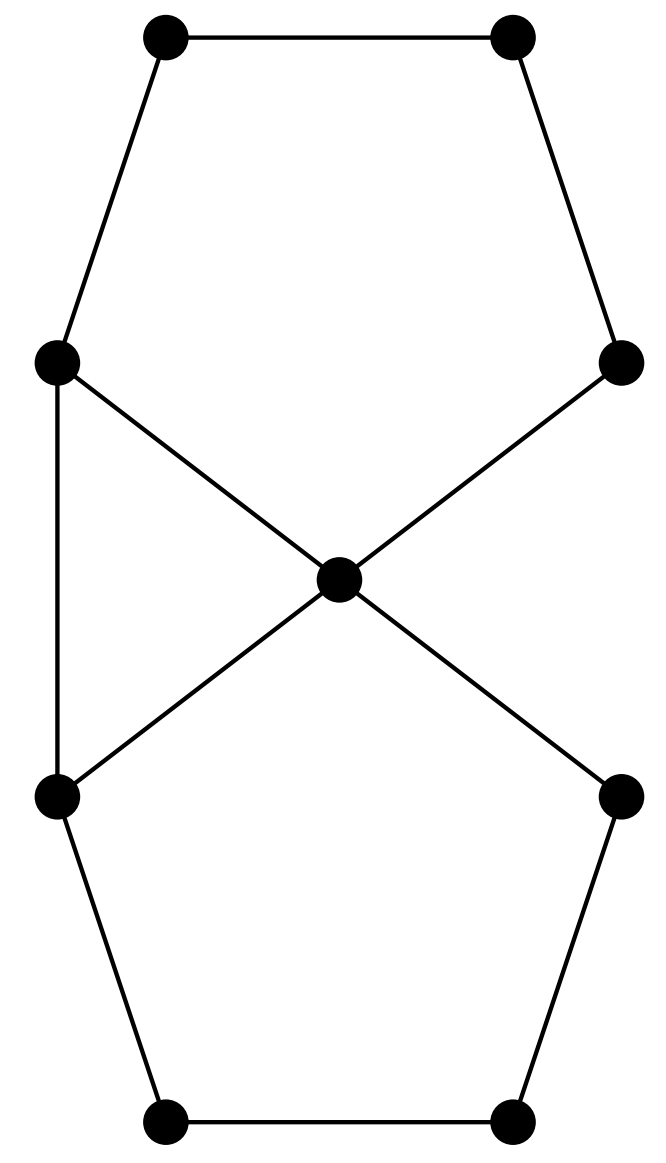


Introduction $(\alpha)$

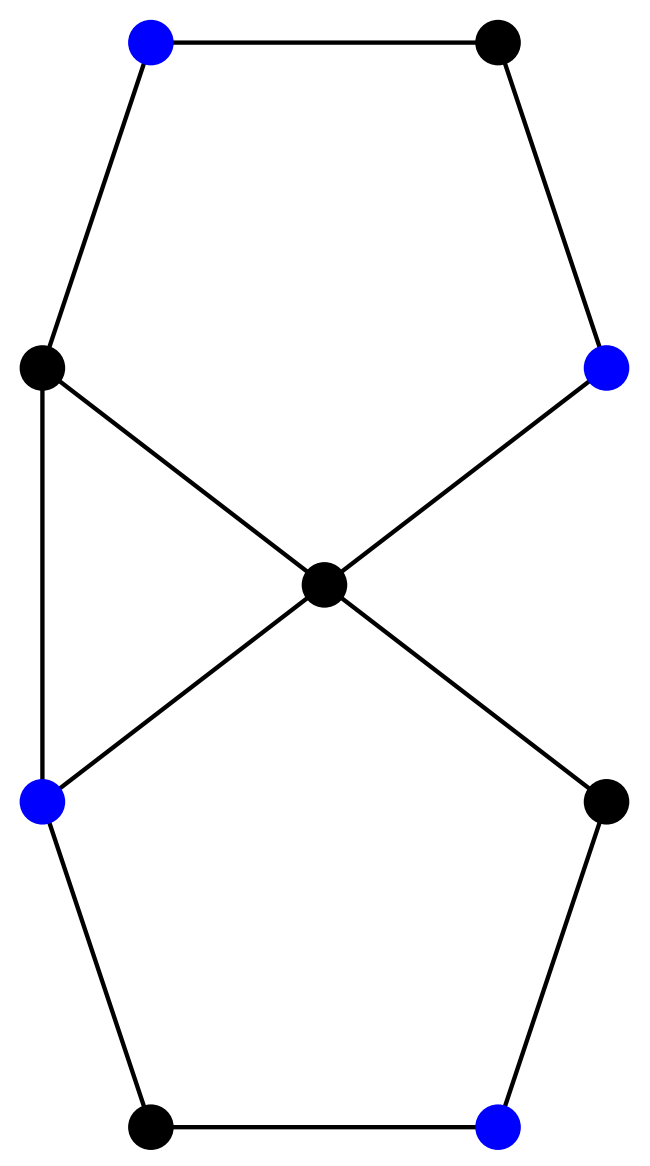




\section{Introduction $(\bar{\chi})$}

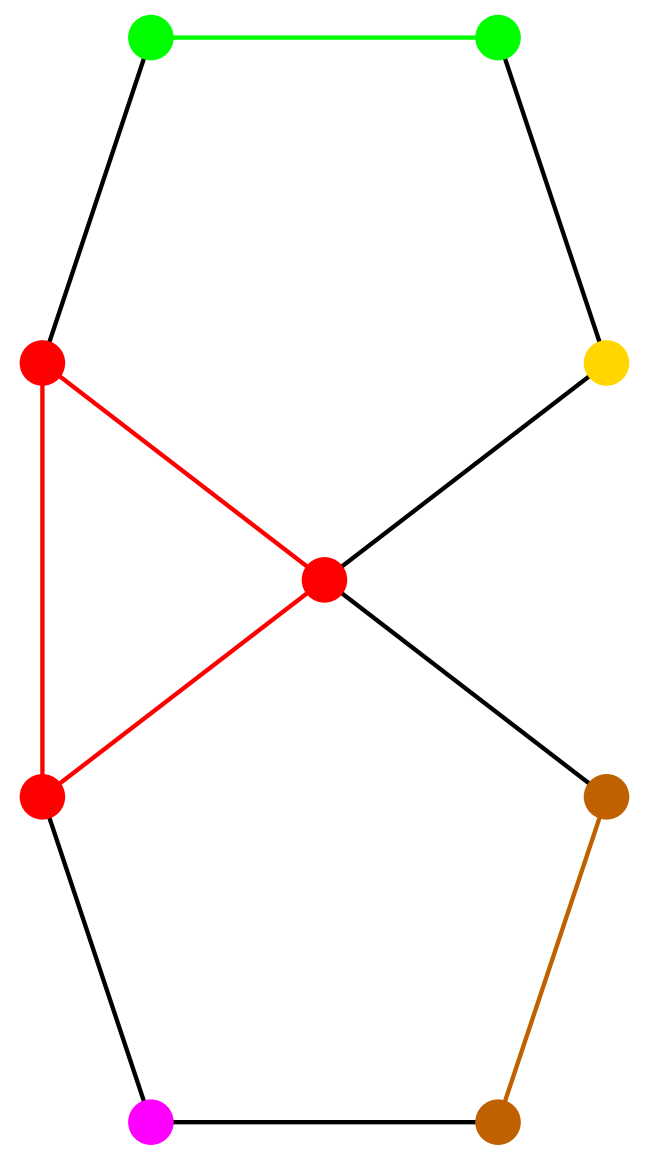




\section{Introduction}

We will study the "sandwich property" of the edge graph introduced in:

D.C., V. Jost: A one-to-one correspondence between colorings and stable sets. Oper. Res.

Lett. 36(6) 673-676 (2008) 


\section{The fractional clique covering number $\bar{\chi}_{f}(G)$}

Let $G=(V, E)$ be a graph with clique-set $\mathcal{K}$.

$$
\begin{aligned}
\alpha(G) & =\max \left\{\sum_{v \in V} x_{v}: \quad \sum_{v \in K} x_{v} \leq 1, \quad \forall K \in \mathcal{K} ; \quad x \in\{0,1\}^{V}\right\} \\
& \leq \max \left\{\sum_{v \in V} x_{v}: \quad \sum_{v \in K} x_{v} \leq 1, \quad \forall K \in \mathcal{K} ; \quad x \geq 0\right\} \\
& =\min \left\{\sum_{K \in \mathcal{K}} y_{K}: \quad \sum_{K \in \mathcal{K}} y_{K} \geq 1, \quad \forall v \in V ; \quad y \geq 0\right\} \\
\leq & \min \left\{\sum_{K \in \mathcal{K}} y_{K}: \quad \sum_{K \in \mathcal{K}} y_{K} \geq 1, \quad \forall v \in V ; \quad y \in\{0,1\}^{\mathcal{K}}\right\} \\
& =\bar{\chi}(G)
\end{aligned}
$$

These three parameters are NP-hard to compute:

$$
\alpha(G) \leq \bar{\chi}_{f}(G) \leq \bar{\chi}(G)
$$




\section{The Lovász theta number $\vartheta(G)$}

Let $G=(V, E)$ be a graph and let $\mathcal{M}_{G}$ be the set of symmetric $V \times V$ matrices, the trace of which is 1 , with $M_{u, v}=0$ for distinct adjacent $u, v$, and which are positive semidefinite.

$$
\begin{aligned}
\alpha(G) & \leq \max \left\{\sum_{u, v \in V} M_{u, v}: M \in \mathcal{M}_{G}\right\} \\
& \leq \bar{\chi}_{f}(G) \\
& \leq \bar{\chi}(G)
\end{aligned}
$$

The Lovász theta number $\vartheta(G)$ can be computed in polynomial time ( $\varepsilon$ approx). 


\section{Example: The 5-cycle}

If $G=C_{5}$, then

$$
\begin{aligned}
\alpha\left(C_{5}\right) & =2 \\
& <\sqrt{5} \\
& =\vartheta\left(C_{5}\right) \\
& <5 / 2 \\
& =\bar{\chi}_{f}\left(C_{5}\right) \\
& <3 \\
& =\bar{\chi}\left(C_{5}\right)
\end{aligned}
$$

$(\sqrt{5} \simeq 2.236)$ 


\title{
Other polynomial sandwich functions
}

\author{
$\vartheta^{\prime}(G)$
}

R.J. McEliece, E.R. Rodemich, H.C. Rumsey Jr.: The Lovász bound and some generalizations, J. Combin. Inform. System Sci. 3 134-152 (1978) and

A. Schrijver: A comparison of the Delsarte and Lovász bounds, IEEE Transactions on Information Theory IT-25 425-429 (1979)

$$
\vartheta^{+}(G)
$$

M. Szegedy: A note on the Theta number of Lovász and the generalized Delsarte bound, FOCS 36-39 (1994)

$$
\vartheta^{+\triangle}(G)
$$

P. Meurdesoif: Strengthening the Lovász Theta(G) bound for graph coloring. Math. Program. 102(3) 577-588 (2005)

$$
\vartheta^{\prime \triangle}(G)
$$

I. Dukanovic, F. Rendl: Semidefinite programming relaxations for graph coloring and maximal clique problems, Math. Program. 109(2-3) 345-365 (2007) 


\section{The $\bar{\chi}_{f}(G)$ barrier}

The polynomial sandwich functions satisfy:

$$
\alpha(G) \leq \vartheta^{\prime} \triangle(G) \leq \vartheta^{\prime}(G) \leq \vartheta(G) \leq \vartheta^{+}(G) \leq \vartheta^{+} \triangle(G) \leq \bar{\chi}_{f}(G) \leq \bar{\chi}(G)
$$

There is no polynomial graph parameter $\beta$ with $\frac{|V(G)|}{\omega(G)} \leq \beta(G) \leq \bar{\chi}(G)$ unless $\mathrm{P}=\mathrm{NP}$.

$\Rightarrow$ no poly $\beta$ with $\bar{\chi}_{f} \leq \beta \leq \bar{\chi}$

N. Gvozdenović and M. Laurent: The operator $\Psi$ for the Chromatic Number of a Graph, SIAM J. Optim., 19(2) 592-615 (2008)

$$
\begin{aligned}
& \Psi: \quad\left[\frac{|V|}{\chi}, \bar{\chi}\right] \quad \rightarrow \quad[\alpha, \bar{\chi}] \\
& {\left[\frac{|V|}{\chi}, \alpha\right] \rightarrow\{\bar{\chi}\}} \\
& {\left[\frac{|V|}{\omega}, \bar{\chi}\right] \quad \rightarrow \quad\{\alpha\}} \\
& \vartheta \quad \mapsto \quad\lceil\vartheta\rceil \\
& \vartheta^{\prime} \quad \mapsto \quad\left\lceil\vartheta^{+}\right\rceil
\end{aligned}
$$




$$
\Psi_{\beta}(G):=\min _{t \in \mathbb{N}} t \quad \text { s.t. } \beta\left(K_{t} \square \bar{G}\right)=|V(G)|
$$
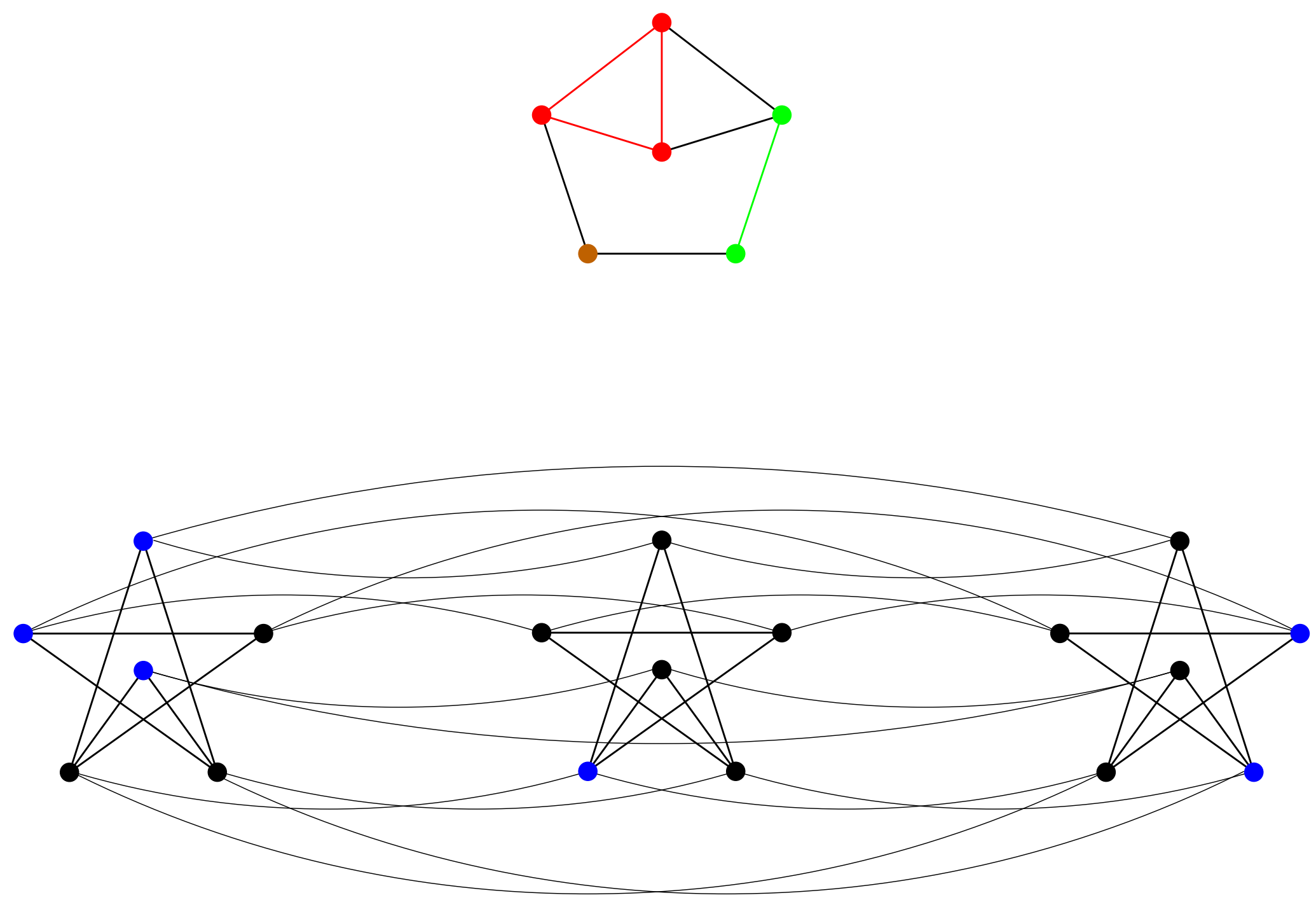


$$
\Psi_{\beta}(G):=\min _{t \in \mathbb{N}} t \quad \text { s.t. } \beta\left(K_{t} \square \bar{G}\right)=|V(G)|
$$
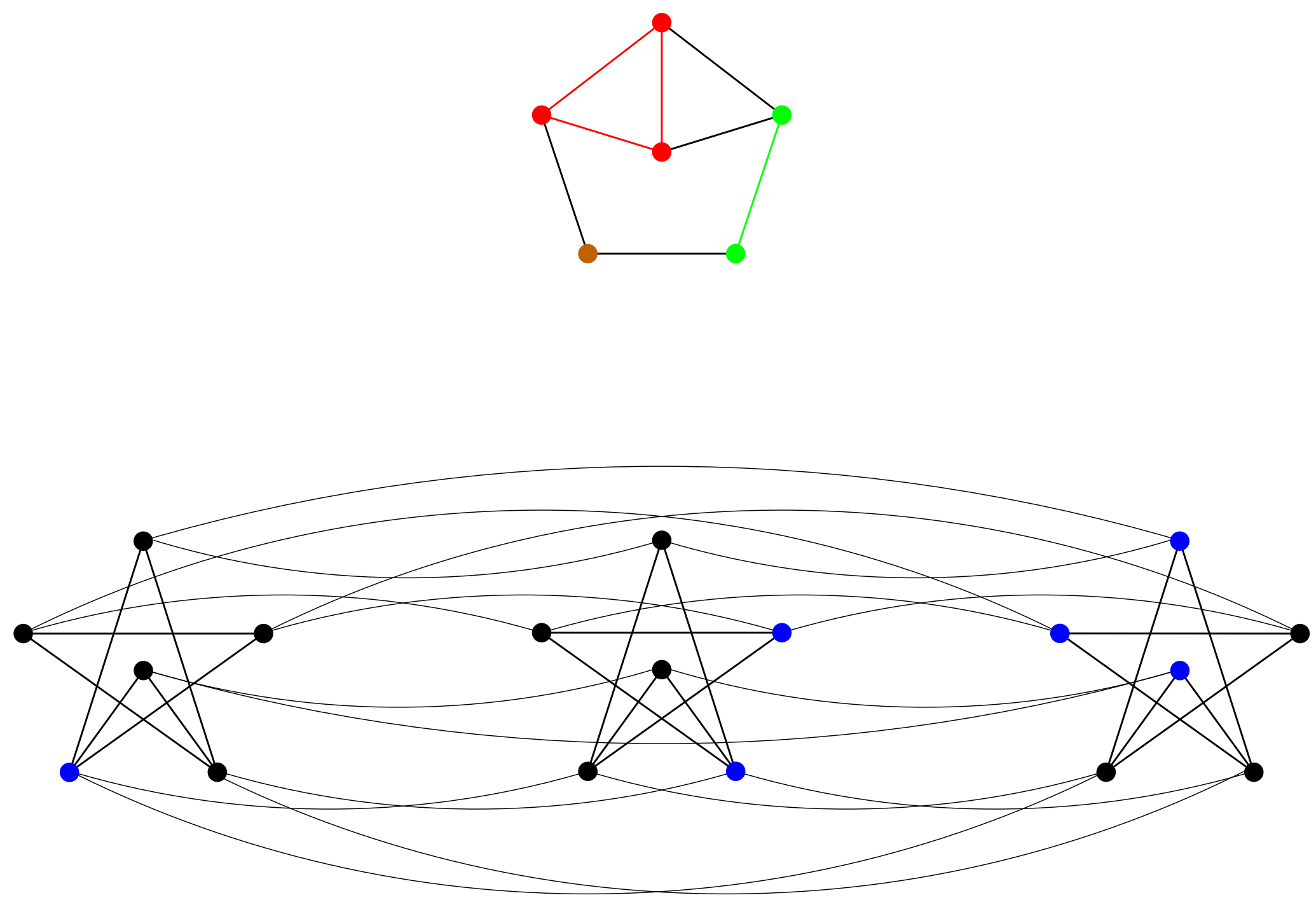


\section{Line graph of triangle free graphs}

The line-graph $L(G)$ of $G$ is the graph with node-set the edge-set of $G$ and where two nodes $e, f$ are nonadjacent in $L(G)$ if they correspond to two disjoint edges of $G$.

If $L(G)$ is the line-graph of a triangle-free $G$, then :

$$
\begin{aligned}
& |V(G)|-\alpha(G)=\bar{\chi}(L(G)) \\
& |V(G)|-\bar{\chi}(G)=\alpha(L(G))
\end{aligned}
$$




\section{Illustrating $|V(G)|-\alpha(G)=\bar{\chi}(L(G))$}
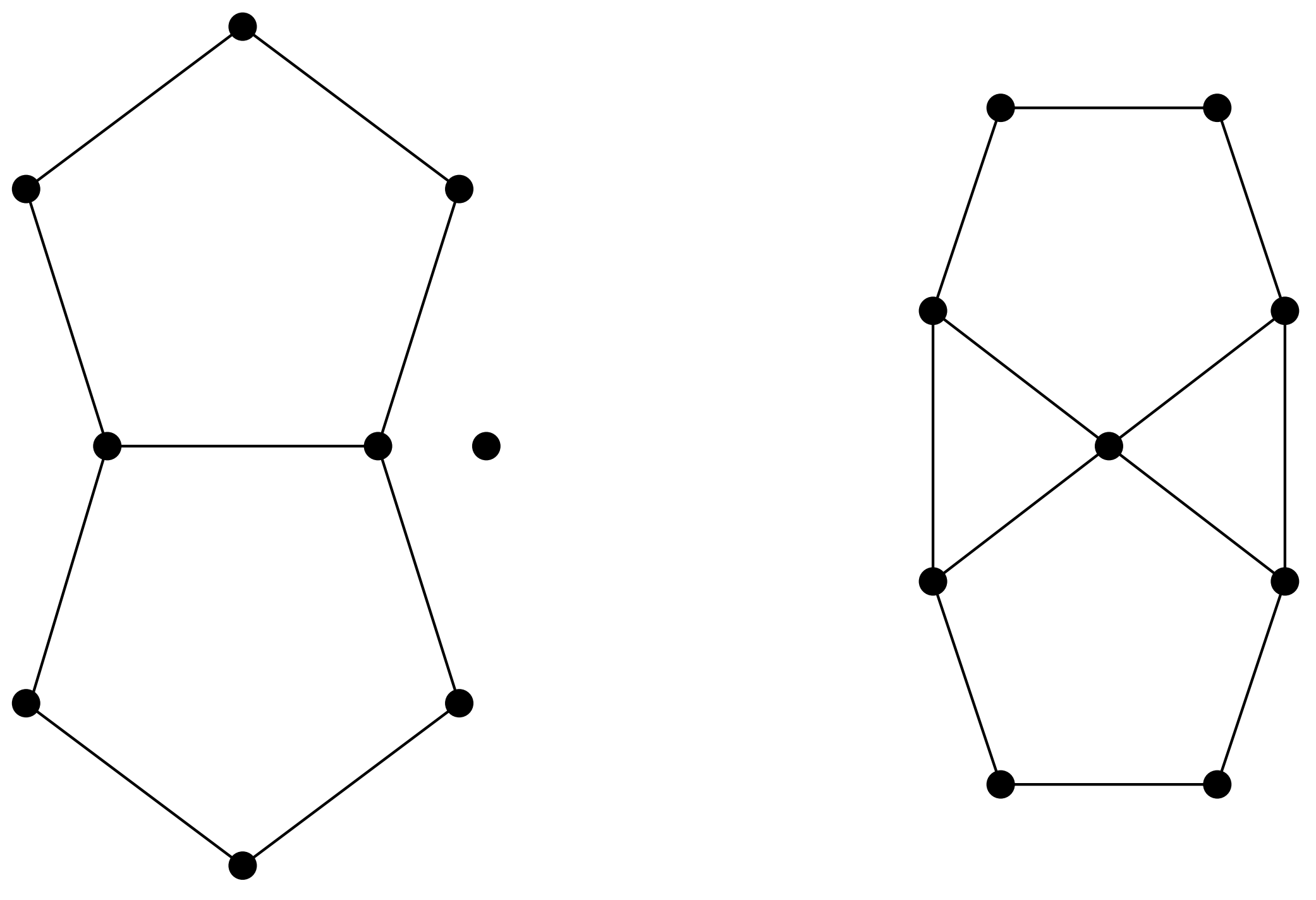


\section{Illustrating $|V(G)|-\alpha(G)=\bar{\chi}(L(G))$}
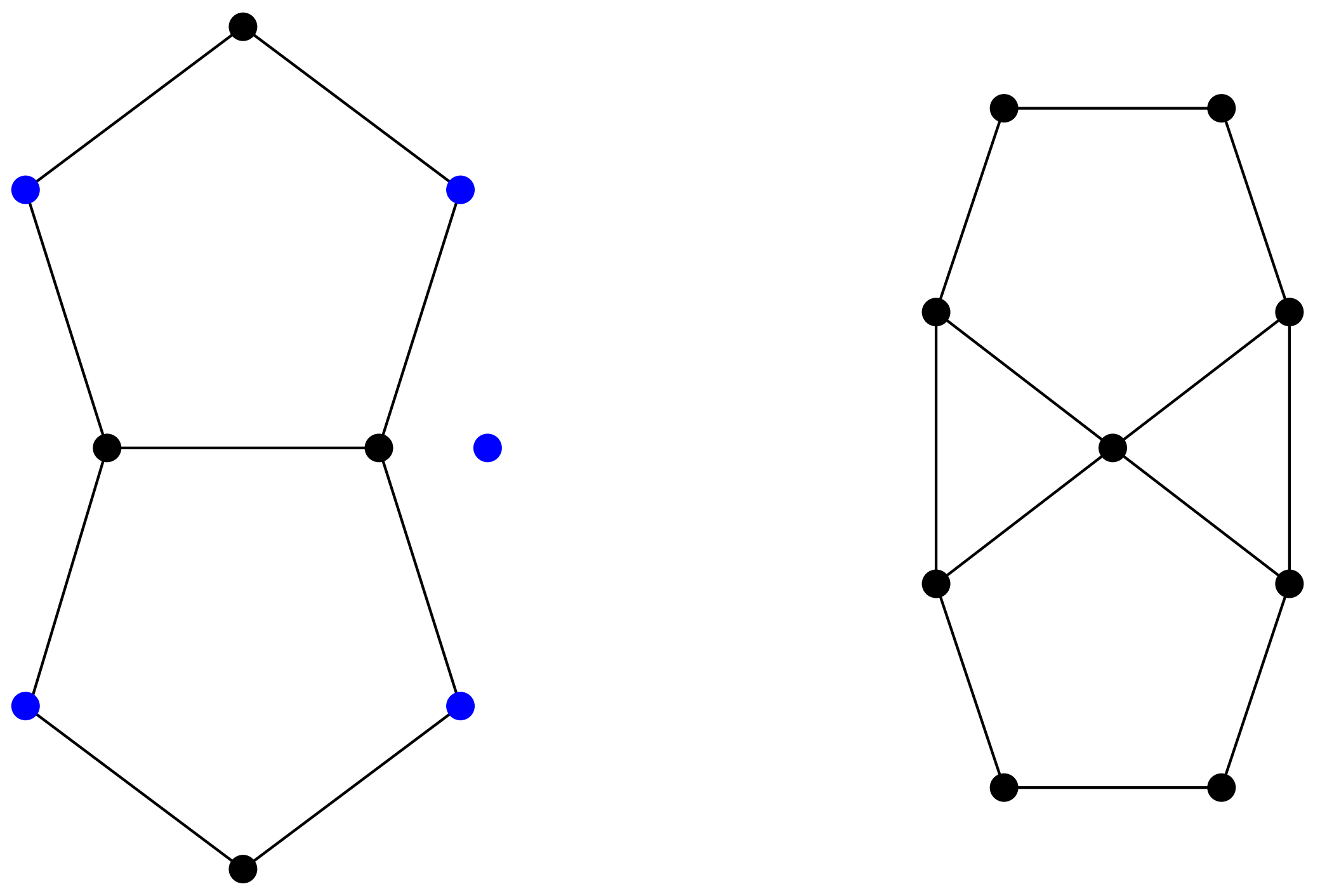


\section{Illustrating $|V(G)|-\alpha(G)=\bar{\chi}(L(G))$}
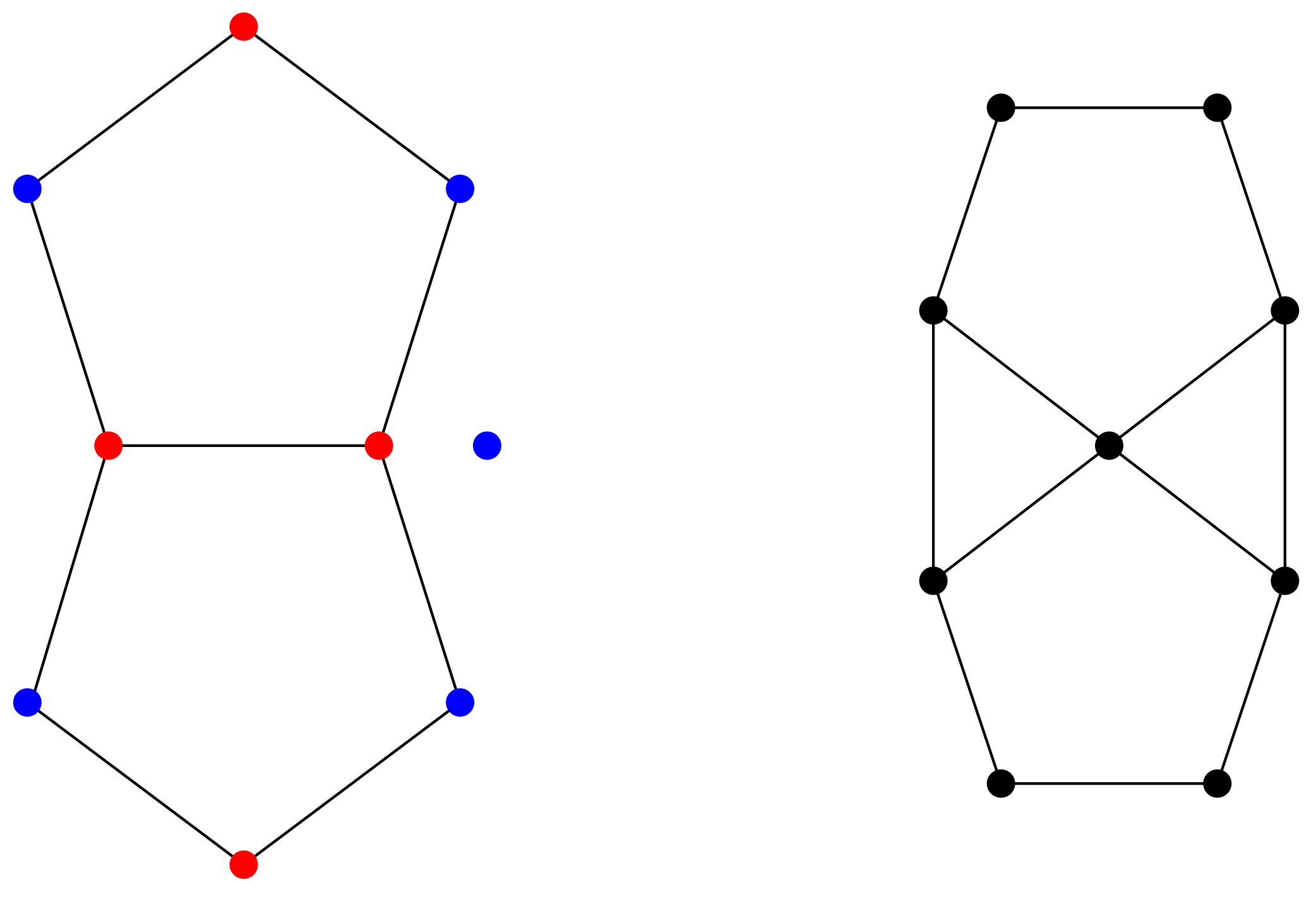


\section{Illustrating $|V(G)|-\alpha(G)=\bar{\chi}(L(G))$}
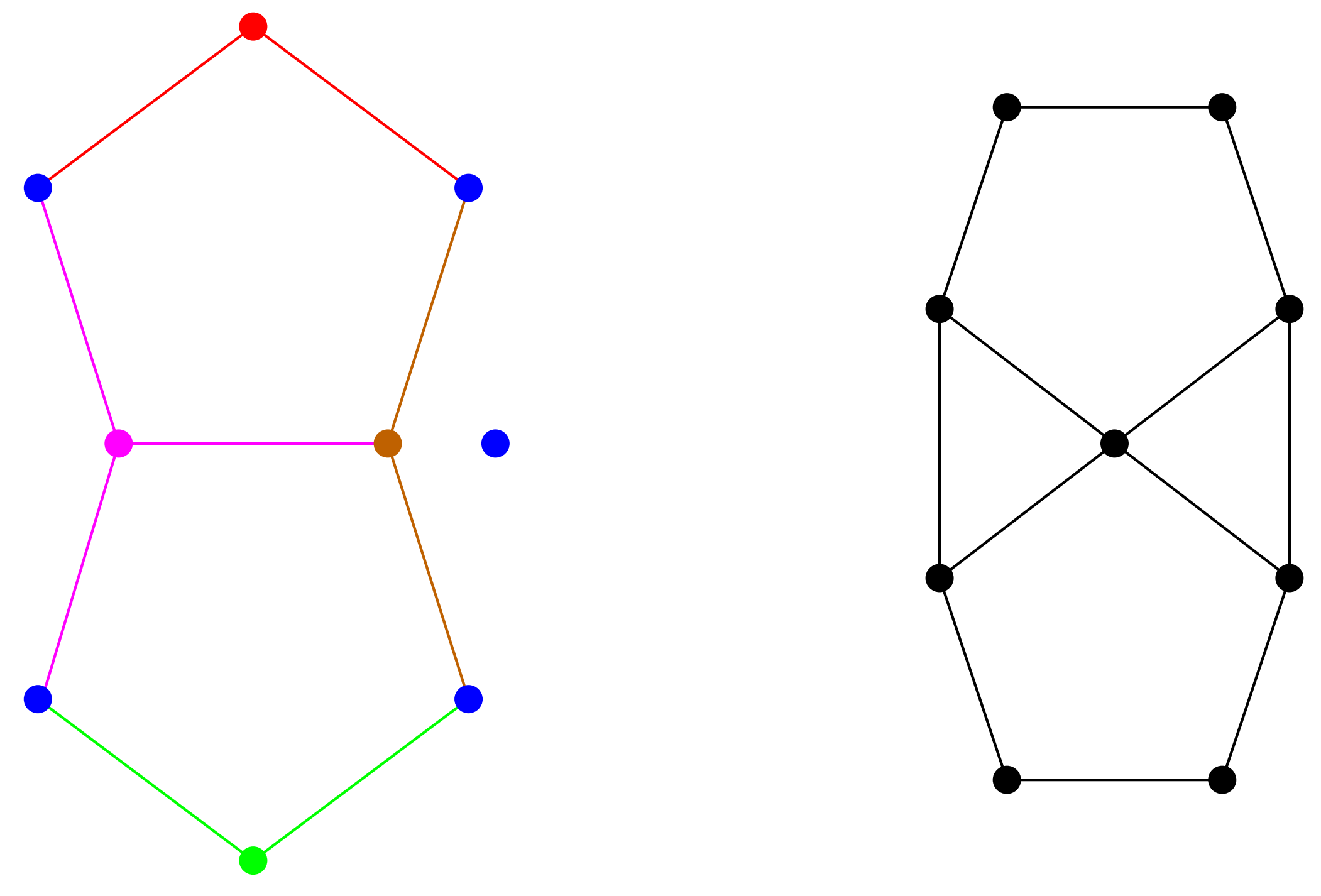


\section{Illustrating $|V(G)|-\alpha(G)=\bar{\chi}(L(G))$}
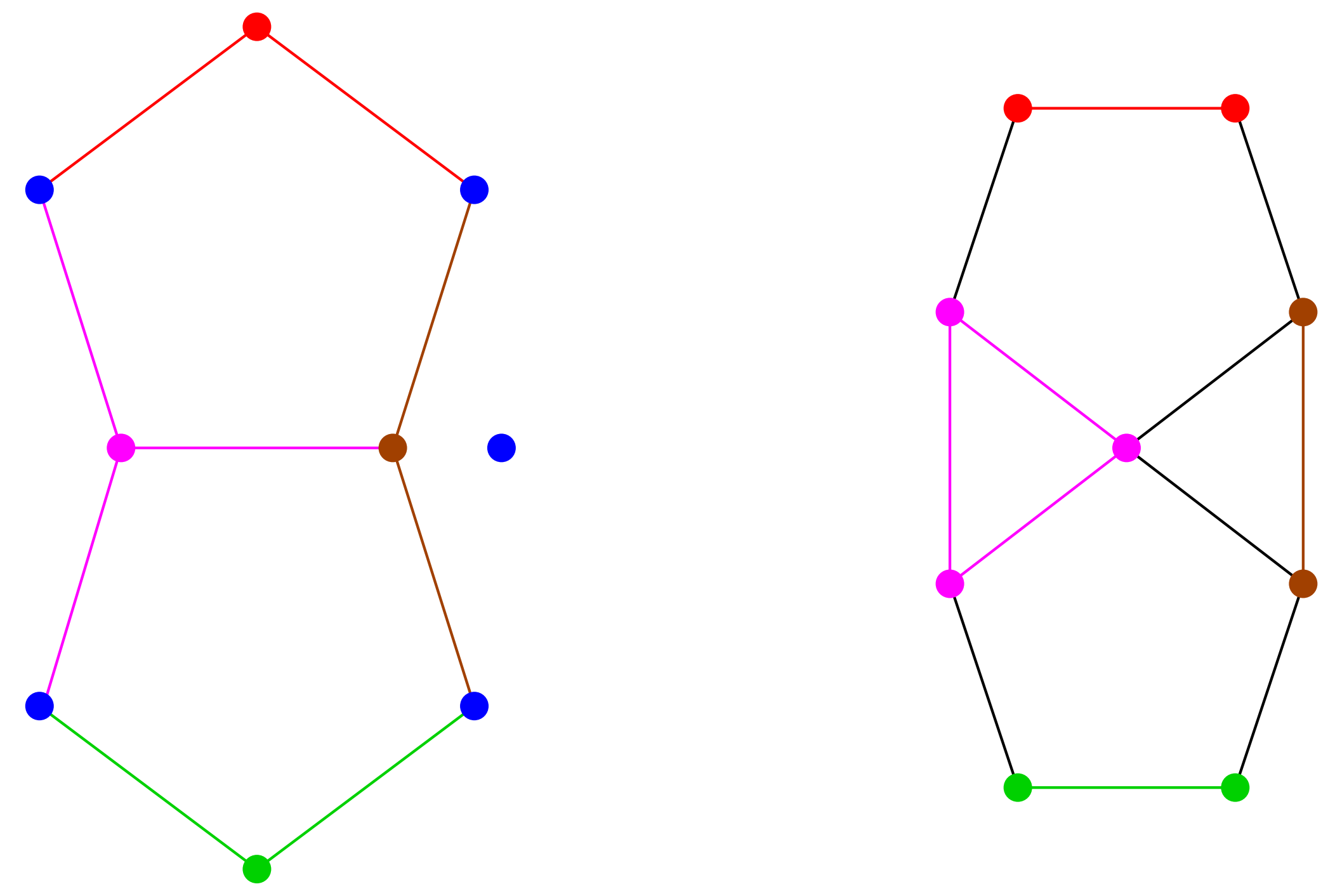


\section{Illustrating $|V(G)|-\bar{\chi}(G)=\alpha(L(G))$}
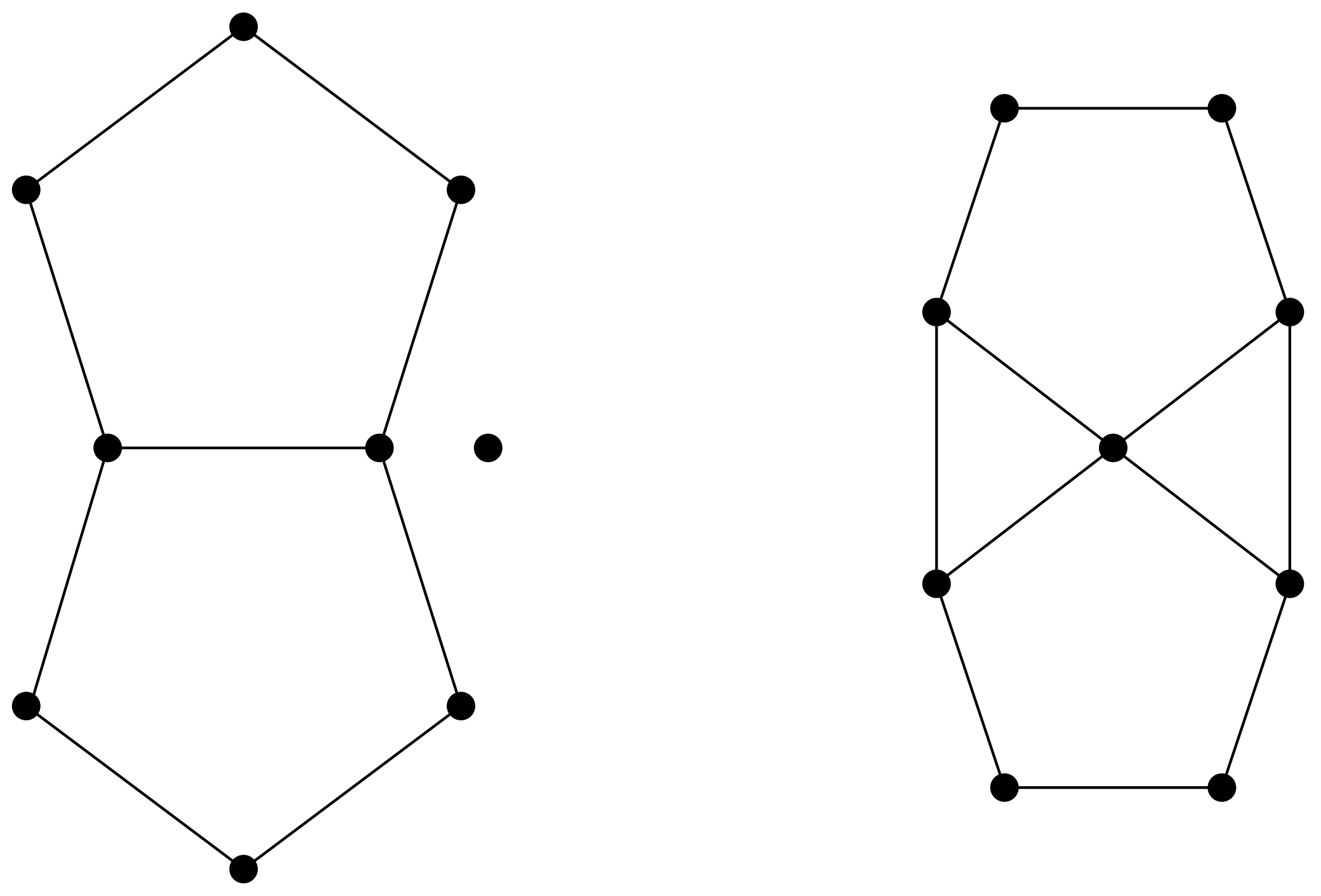


\section{Illustrating $|V(G)|-\bar{\chi}(G)=\alpha(L(G))$}
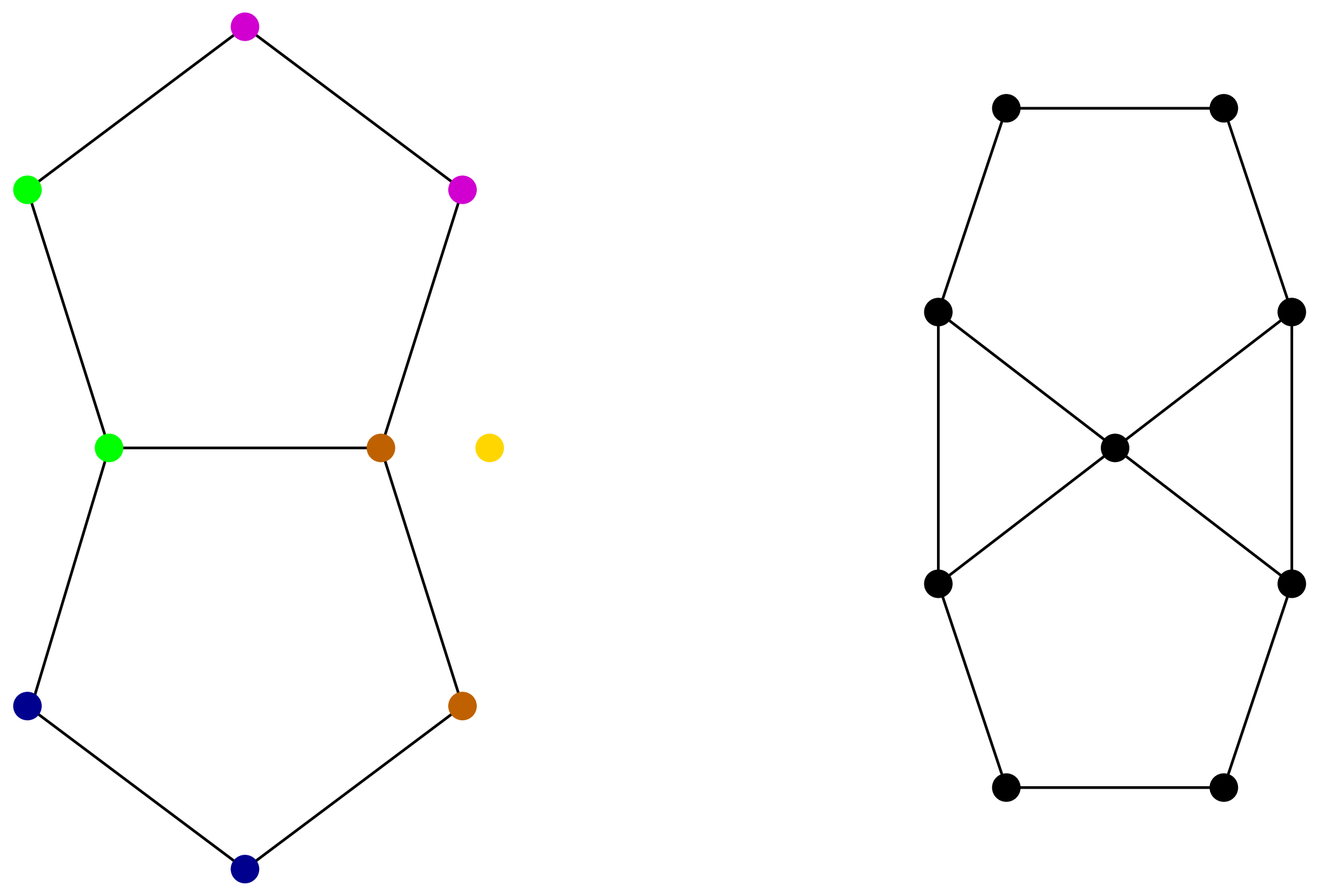


\section{Illustrating $|V(G)|-\bar{\chi}(G)=\alpha(L(G))$}
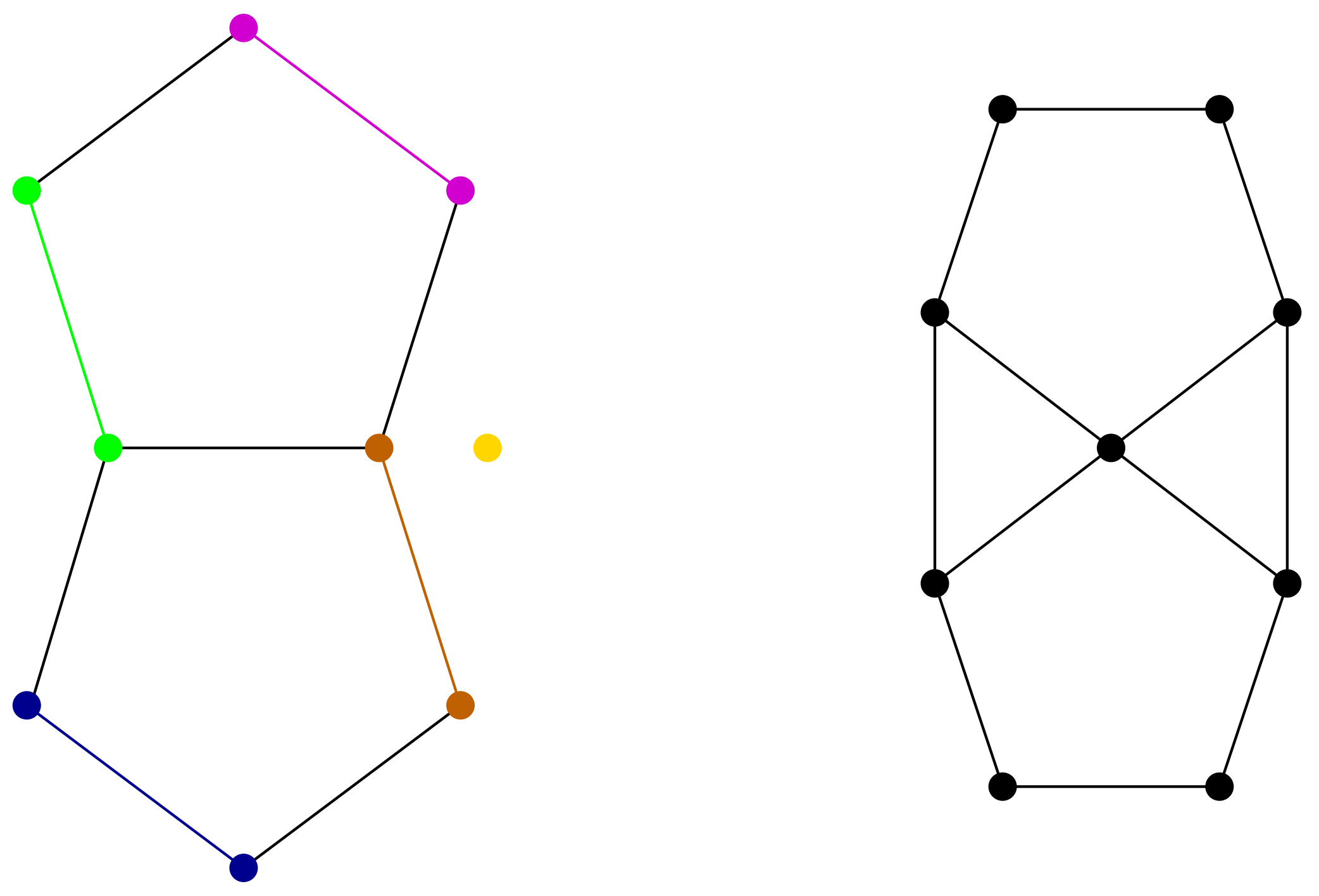


\section{Illustrating $|V(G)|-\bar{\chi}(G)=\alpha(L(G))$}
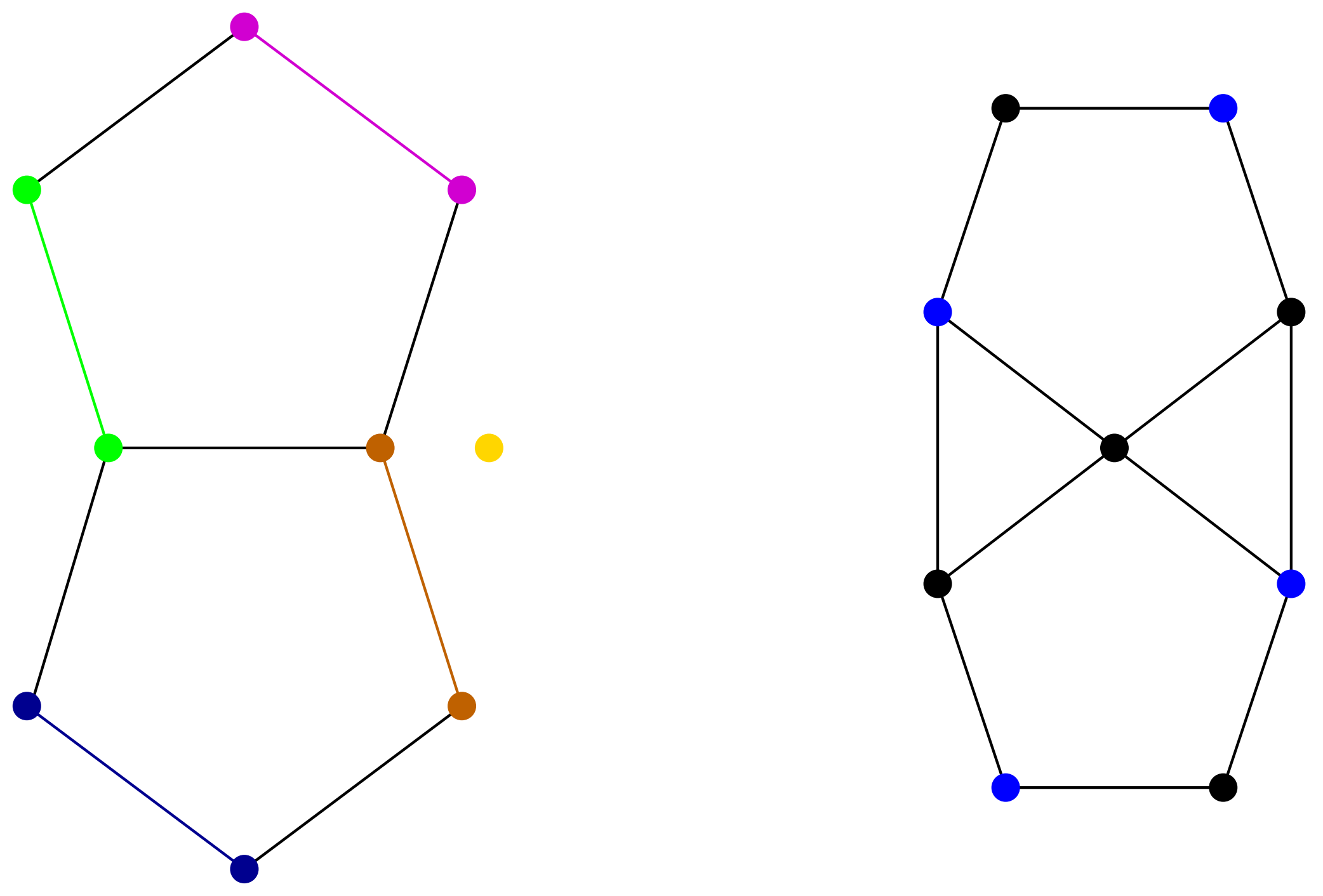


\section{Line graph of triangle free graphs}

If $L(G)$ is the line-graph of a triangle-free $G$, then :

(P1) $|V(G)|-\alpha(G)=\bar{\chi}(L(G))$;

(P2) $|V(G)|-\bar{\chi}(G)=\alpha(L(G))$; 


\section{Line graph of triangle free graphs}

If $L(G)$ is the line-graph of a triangle-free $G$, then :

(P1) $|V(G)|-\alpha(G)=\bar{\chi}(L(G))$;

(P2) $|V(G)|-\bar{\chi}(G)=\alpha(L(G))$;

and it follows from (P1)-(P2) that

(P3) If $\alpha \leq \beta \leq \bar{\chi}(\forall G)$ and if $G$ is triangle-free then,

$$
\alpha(G) \leq|V(G)|-\beta(L(G)) \leq \bar{\chi}(G) .
$$




\section{Line graph of triangle free graphs}

If $L(G)$ is the line-graph of a triangle-free $G$, then :

(P1) $|V(G)|-\alpha(G)=\bar{\chi}(L(G))$;

(P2) $|V(G)|-\bar{\chi}(G)=\alpha(L(G))$;

and it follows from (P1)-(P2) that

(P3) If $\alpha \leq \beta \leq \bar{\chi}(\forall G)$ and if $G$ is triangle-free then,

$$
\alpha(G) \leq|V(G)|-\beta(L(G)) \leq \bar{\chi}(G) .
$$

$\alpha \leq \beta \Rightarrow|V(G)|-\beta(L(G)) \leq|V(G)|-\alpha(L(G))=\bar{\chi}(G)$. 


\section{Line graph of triangle free graphs}

If $L(G)$ is the line-graph of a triangle-free $G$, then :

(P1) $|V(G)|-\alpha(G)=\bar{\chi}(L(G))$;

(P2) $|V(G)|-\bar{\chi}(G)=\alpha(L(G))$;

and it follows from (P1)-(P2) that

(P3) If $\alpha \leq \beta \leq \bar{\chi}(\forall G)$ and if $G$ is triangle-free then,

$$
\alpha(G) \leq|V(G)|-\beta(L(G)) \leq \bar{\chi}(G) .
$$

$\alpha \leq \beta \Rightarrow|V(G)|-\beta(L(G)) \leq|V(G)|-\alpha(L(G))=\bar{\chi}(G)$.

$\beta \leq \bar{\chi} \quad \Rightarrow \quad \alpha(G)=|V(G)|-\bar{\chi}(L(G)) \leq|V(G)|-\beta(L(G))$. 


\section{Sandwich line graphs}

A sandwich line graph $S(G)$ of $G$ is an edge graph of $G$ such that:

(i) $S(G)=L(G)$ if $G$ is triangle free;

(ii) $S(G)$ satisfies (P1), (P2) and (P3) for any graph $G$. 
Now $G$ has triangle
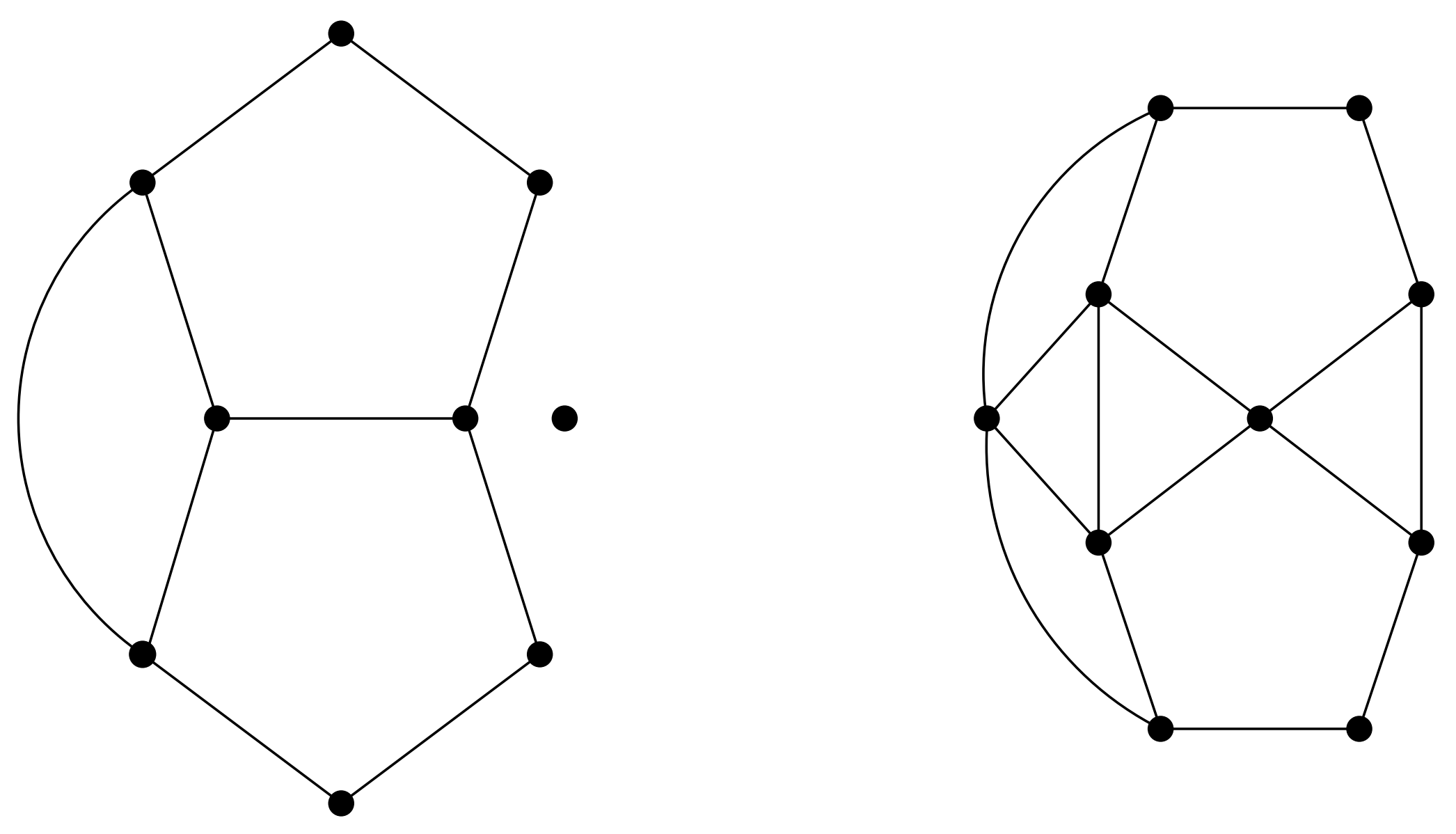
Now $G$ has triangle
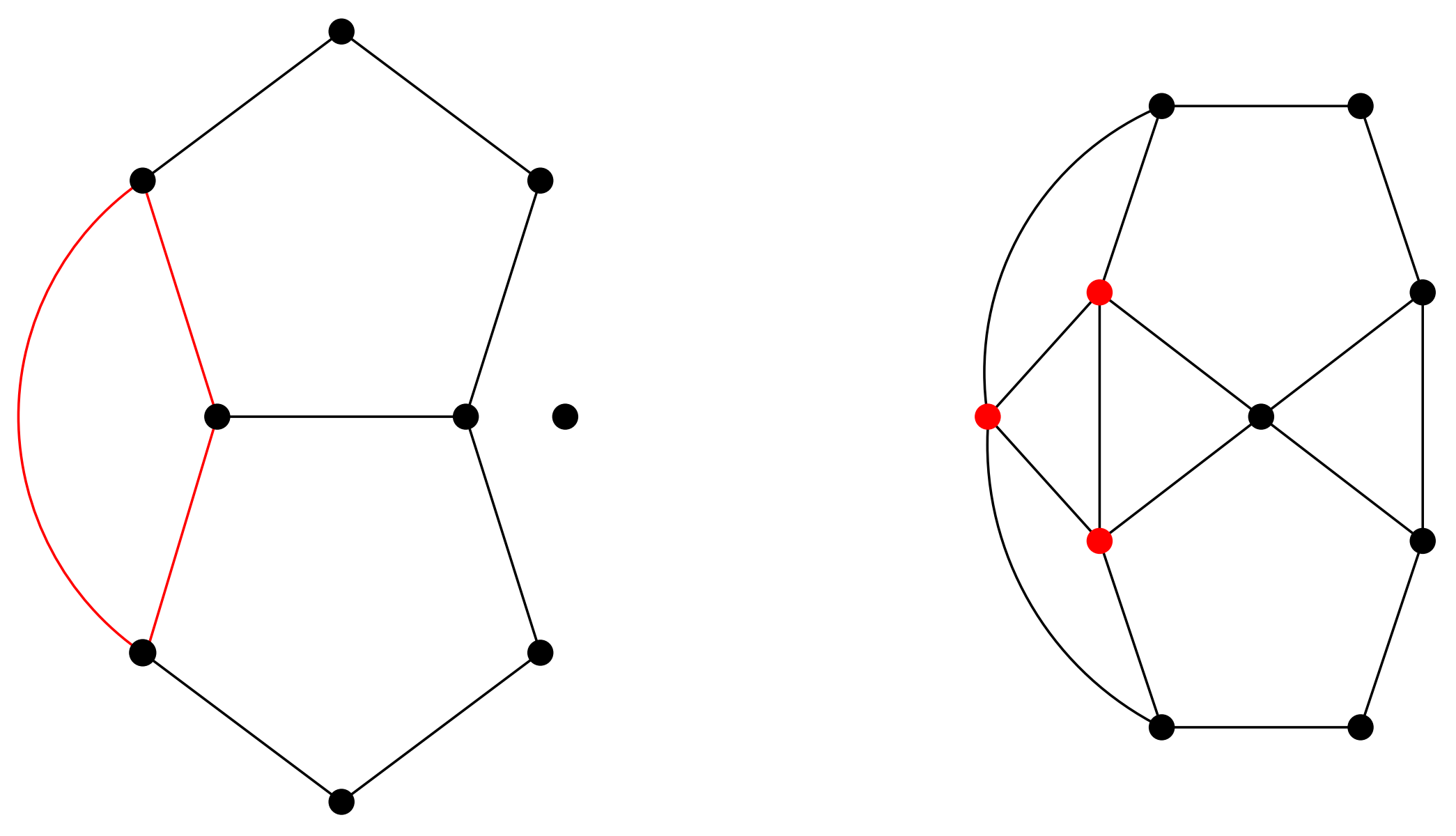
Restoring the sandwich property
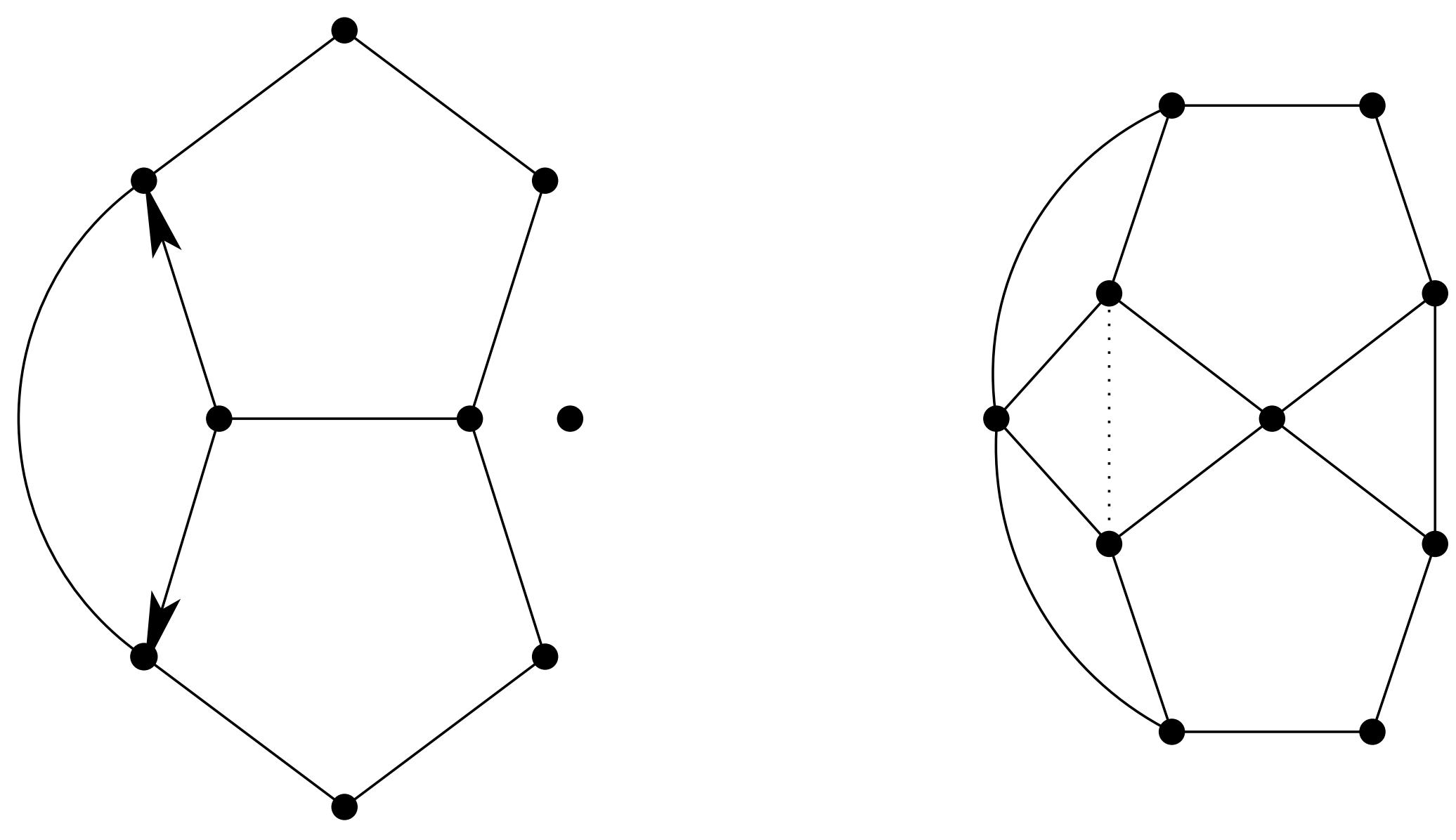
Restoring the sandwich property
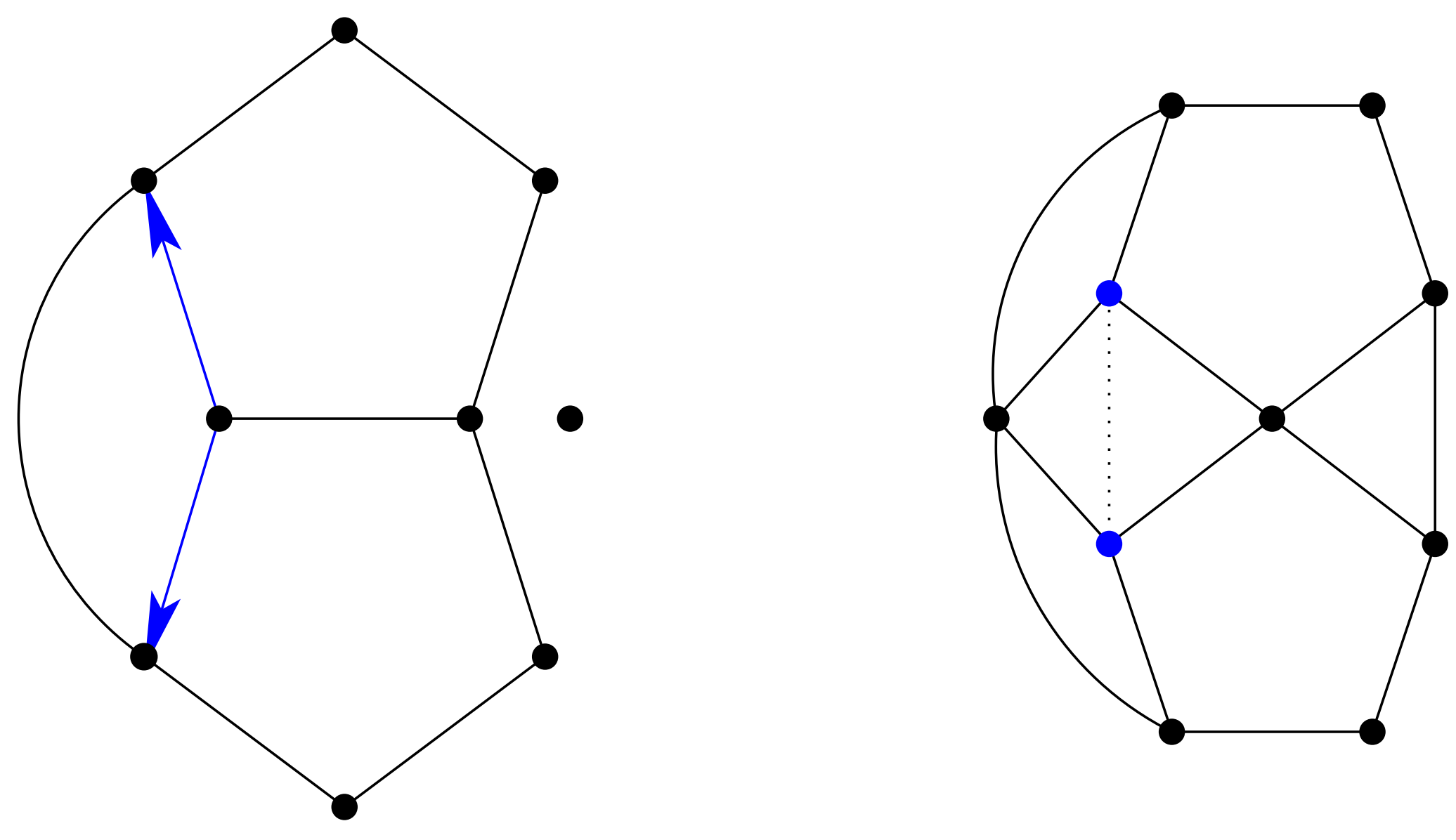
Restoring the sandwich property
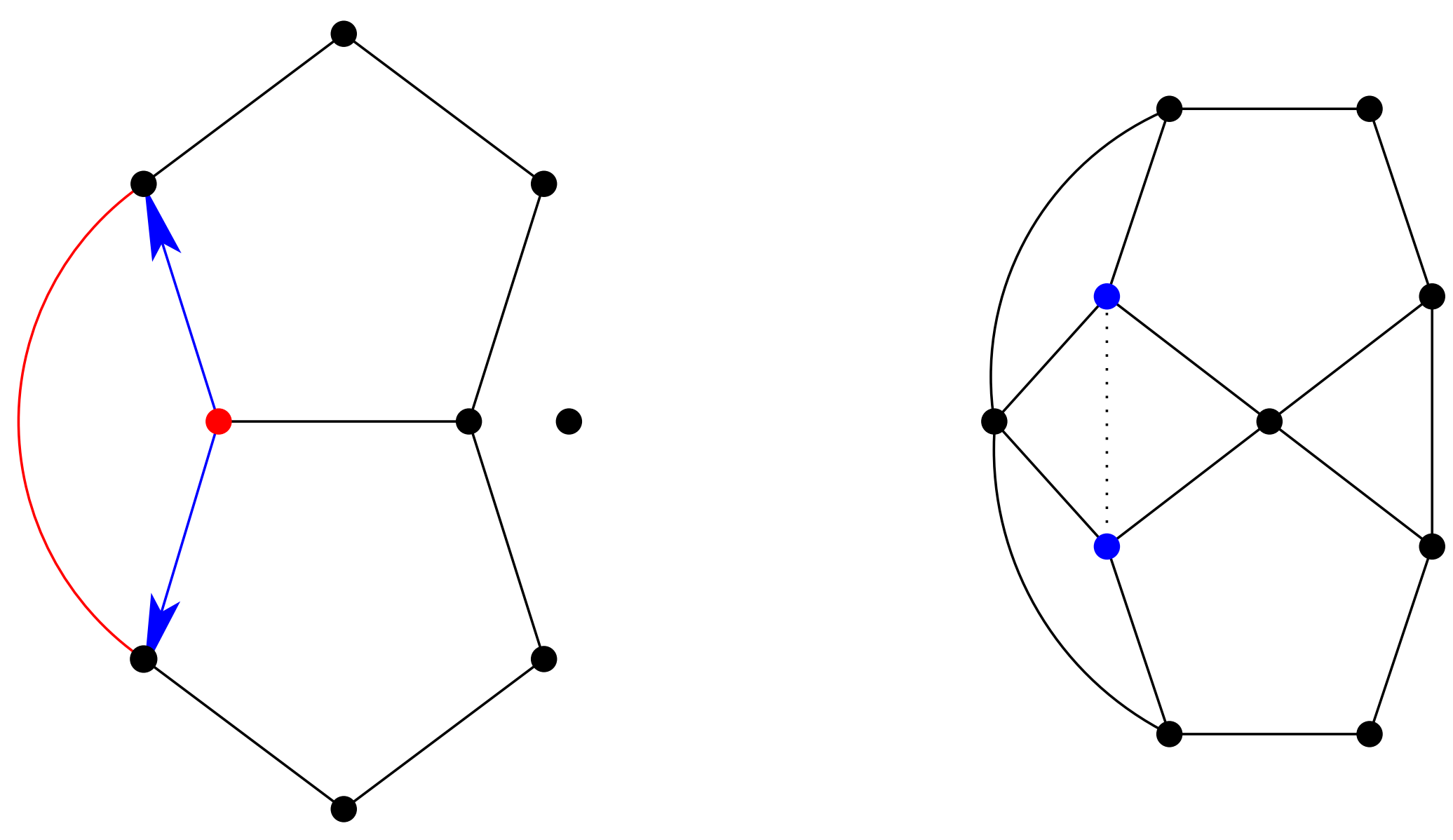
A sandwich line graph $S(G)$ of $G$
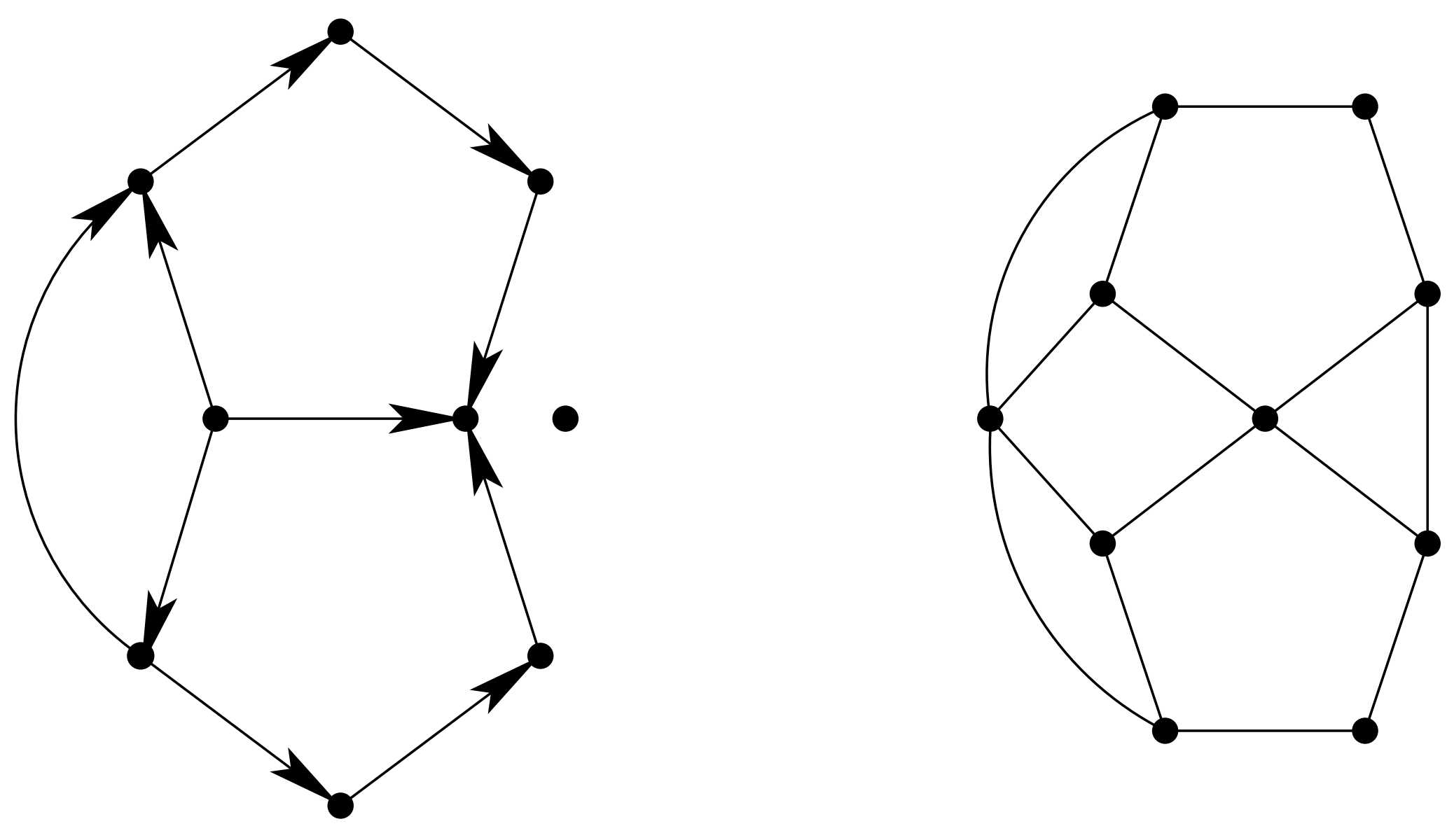


\section{Illustrating $|V(G)|-\alpha(G) \geq \bar{\chi}(S(G))$}
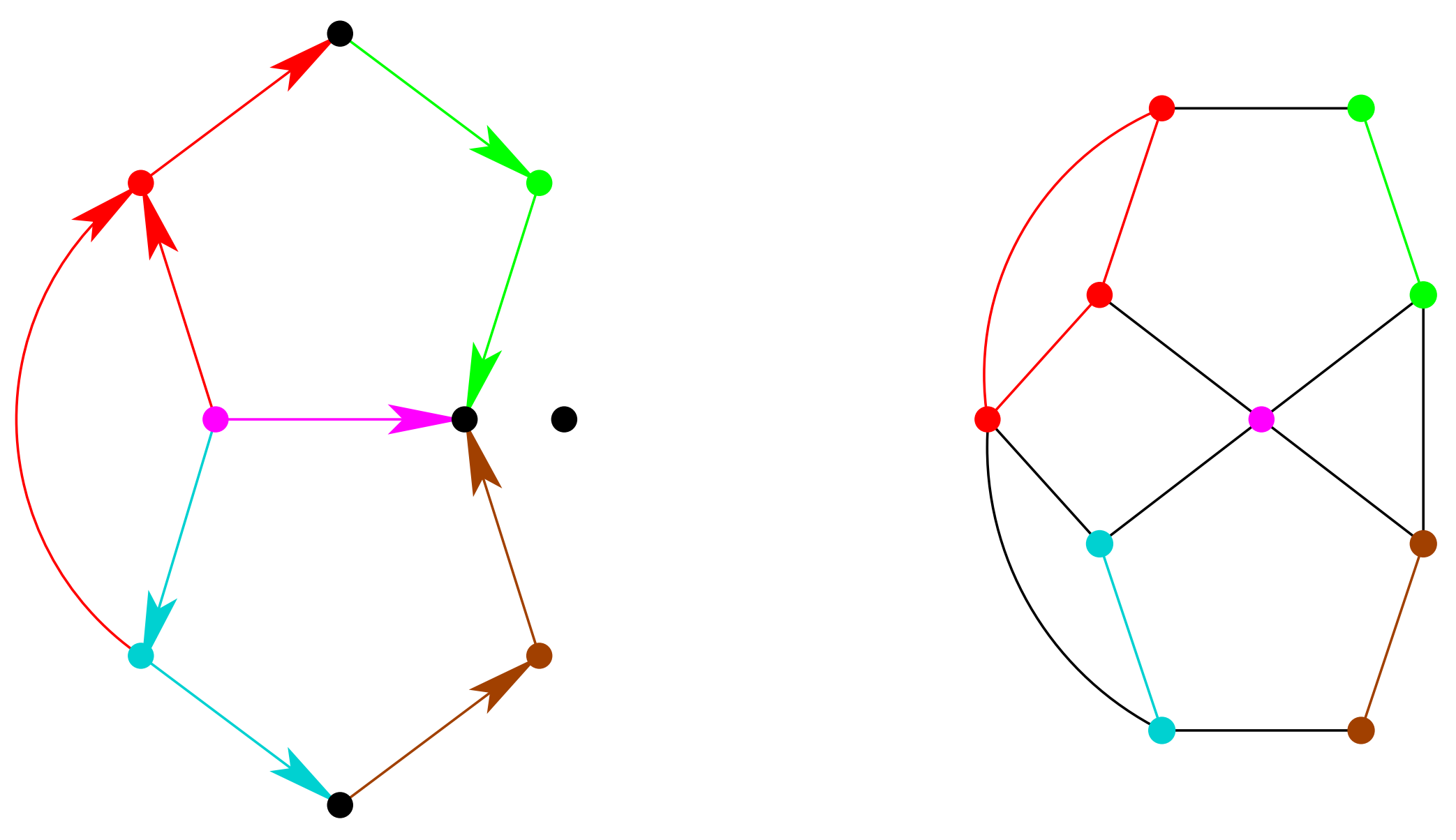


\section{Illustrating $|V(G)|-\alpha(G) \leq \bar{\chi}(S(G))$}
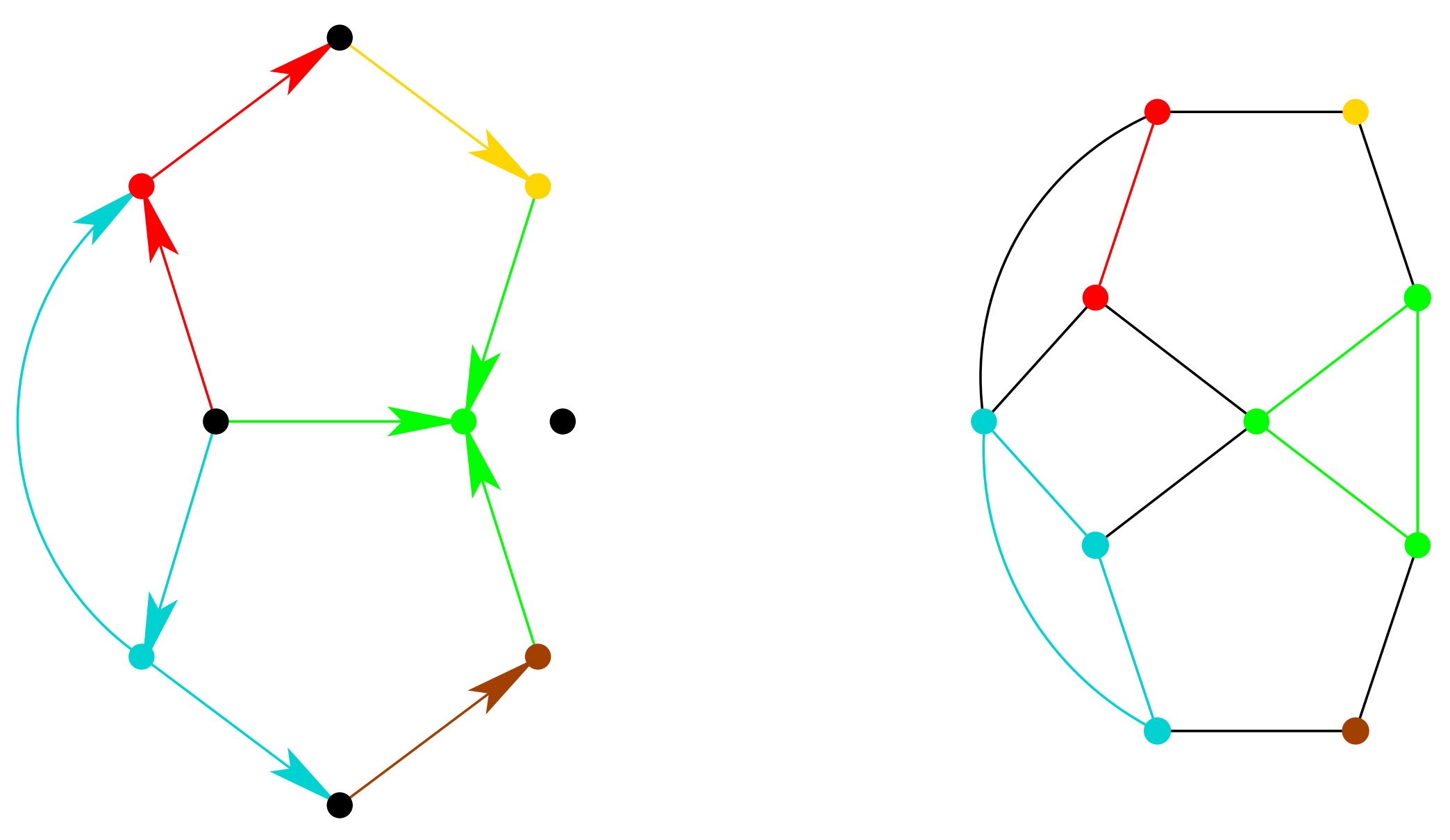


\section{Illustrating $|V(G)|-\bar{\chi}(G) \geq \alpha(S(G))$}
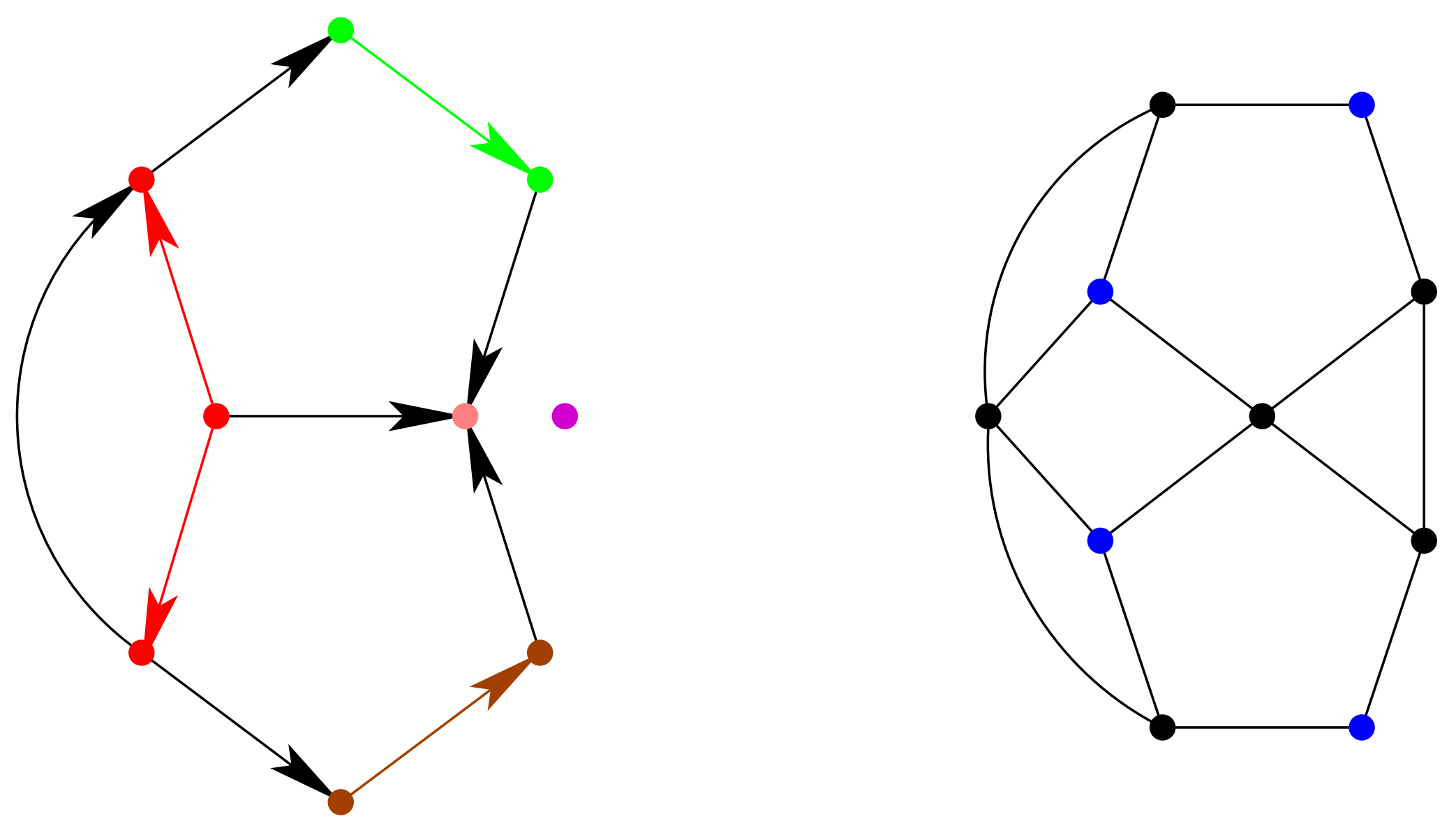


\section{Illustrating $|V(G)|-\bar{\chi}(G) \geq \alpha(S(G))$}
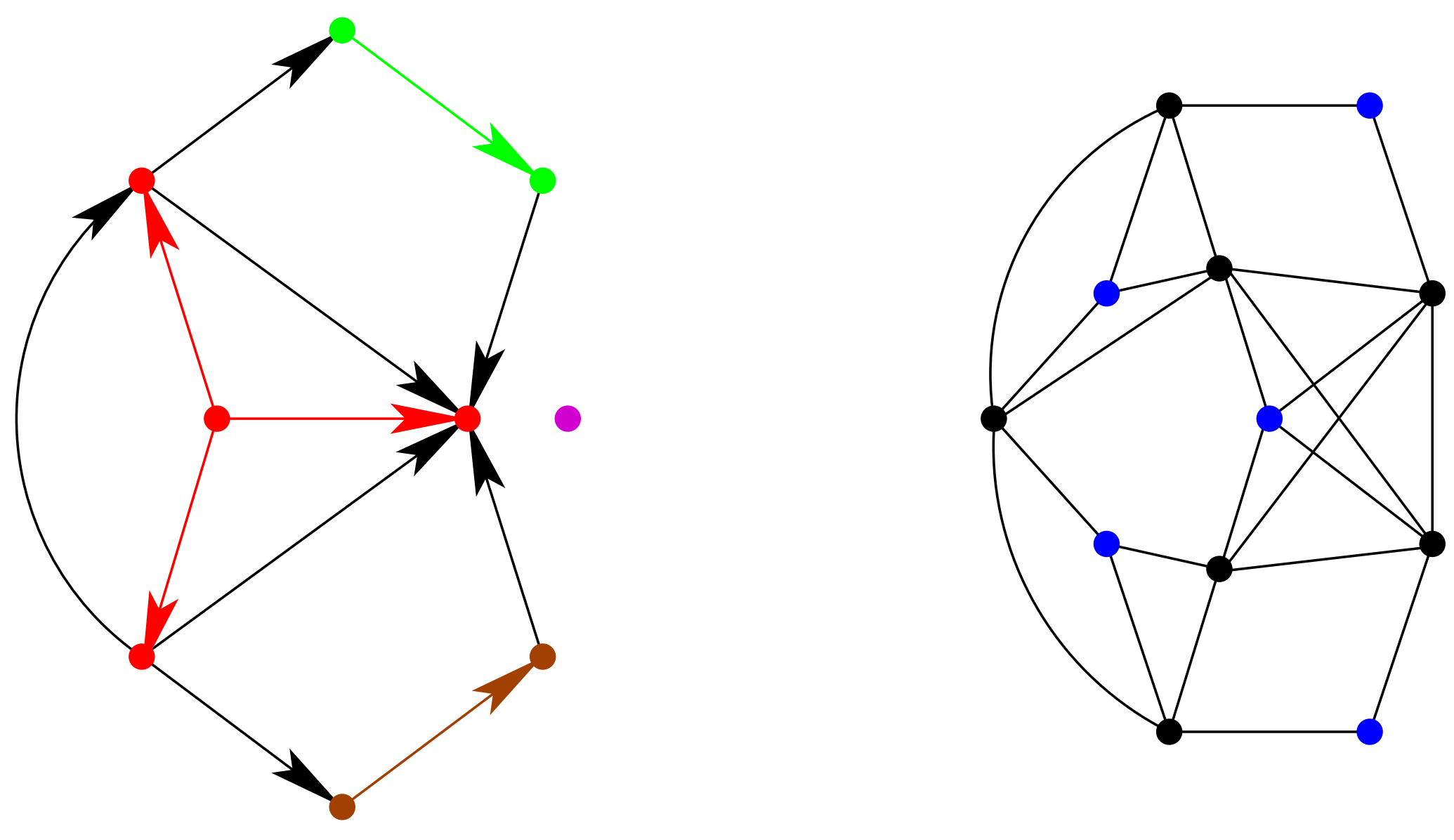


\section{Illustrating $|V(G)|-\bar{\chi}(G) \leq \alpha(S(G))$}
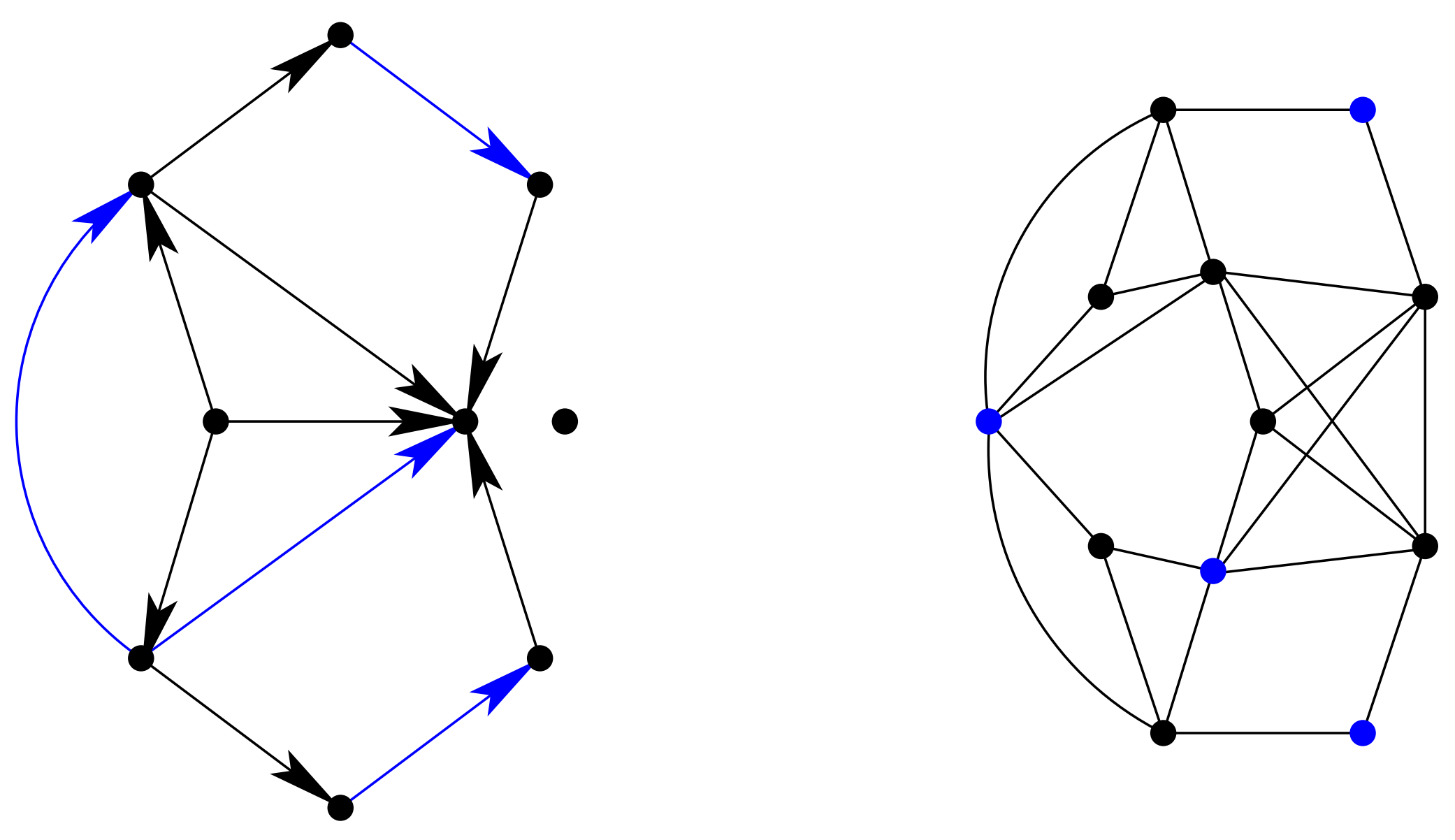


\section{Illustrating $|V(G)|-\bar{\chi}(G) \leq \alpha(S(G))$}
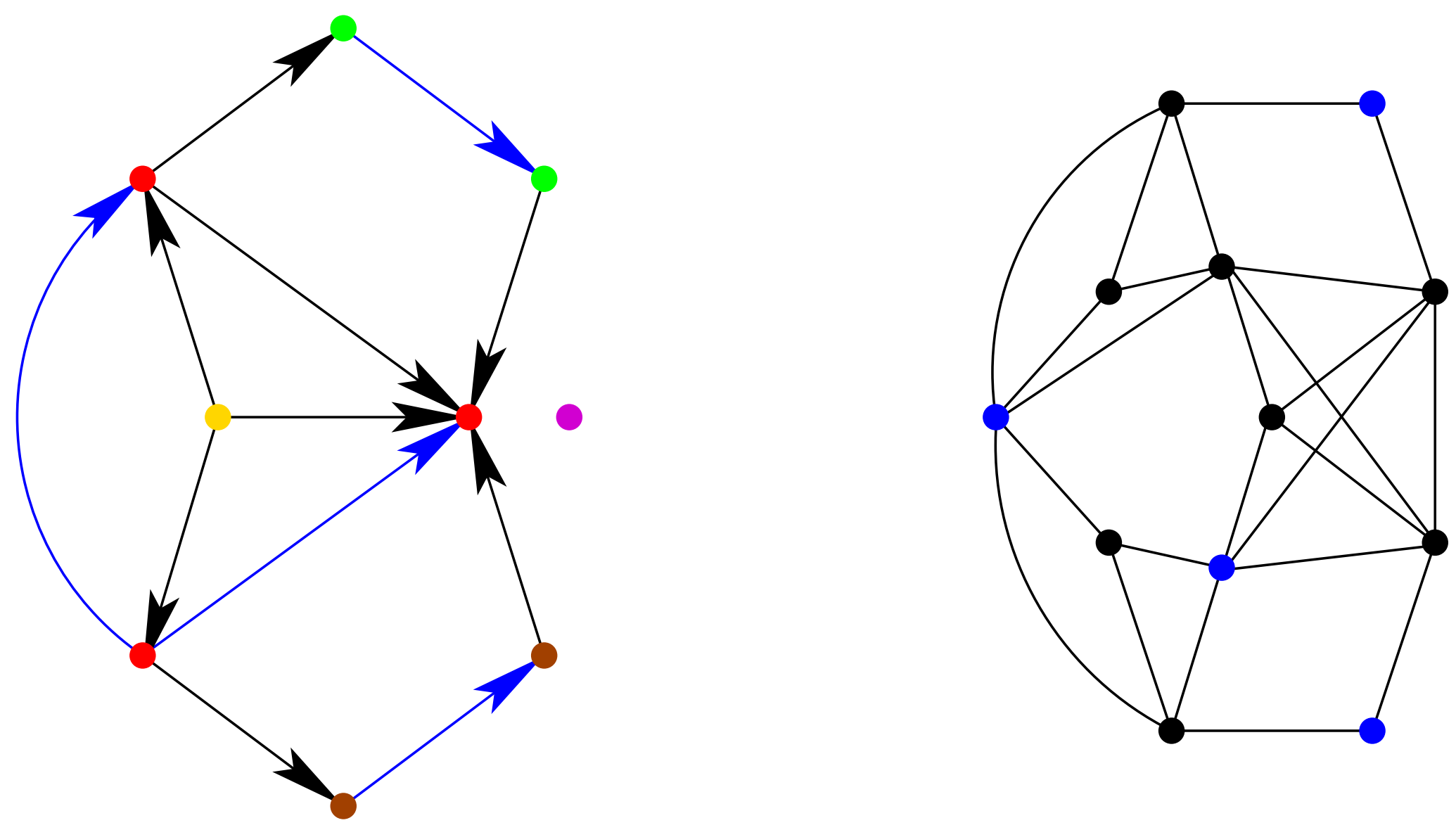


\section{Illustrating $|V(G)|-\bar{\chi}(G) \leq \alpha(S(G))$}
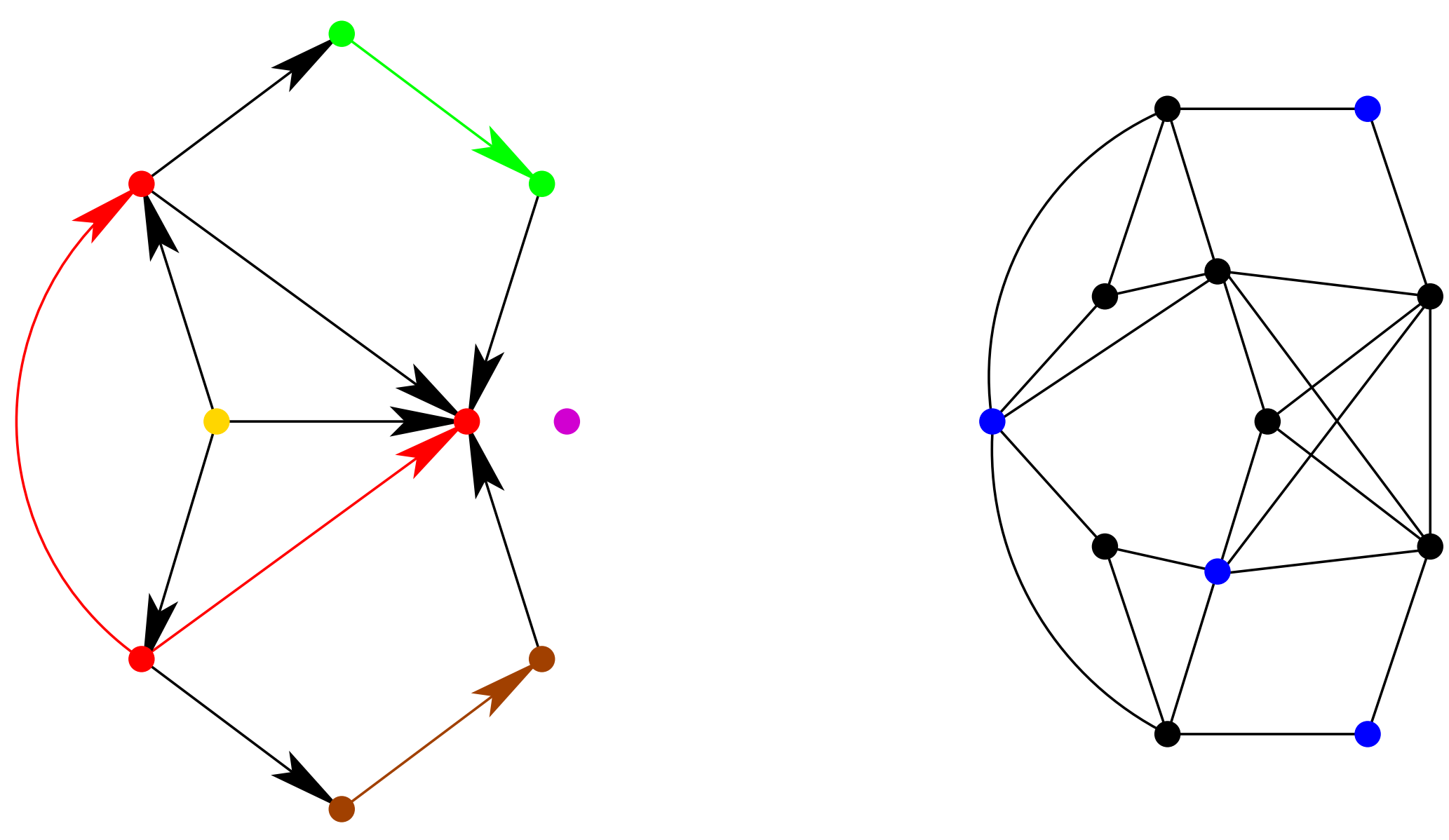


\section{Theorem}

Let $\beta(G)$ be a graph parameter with the following sandwich property:

$$
\alpha(G) \leq \beta(G) \leq \bar{\chi}(G)
$$

for every graph $G$.

Let $G=(V, E)$ be a simple graph.

Let $\sigma: V \leftrightarrow\{1,2, \ldots,|V|\}$ and let $S\left(G_{\sigma}\right)$ be the graph with node-set the edge-set of $G$ and where two nodes $e, f$ are nonadjacent in $S\left(G_{\sigma}\right)$ iff they are disjoint edges in $G$ or $e=u v, f=u w$ but $v w \in E$ and $\sigma(u)<\min \{\sigma(v), \sigma(w)\}$.

Then

$$
\alpha(G) \leq|V(G)|-\beta\left(S\left(G_{\sigma}\right)\right) \leq \bar{\chi}(G), \quad \forall \sigma
$$

futhermore, if $\beta(G)=\bar{\chi}(G)$, then the left inequality holds with equality; and if $\beta(G)=\alpha(G)$, then the right inequality holds with equality. 


\section{A corollary}

Denote $S^{0}(G)=G$ and let $S^{i+1}(G)$ be a sandwich line graph of $S^{i}(G)$.

Thus, for any sandwich function $\beta$ and any integer $k \geq 1$, one has

$$
\alpha(G) \leq(-1)^{k} \beta\left(S^{k}(G)\right)-\sum_{i=0}^{i=k-1}(-1)^{i+1}\left|V\left(S^{i}(G)\right)\right| \leq \bar{\chi}(G)
$$




\section{Particular example : numerical values with $G=k C_{5}$}

\begin{tabular}{c||c|c|c|c|c|c}
$k$ & $\alpha=\Psi_{\bar{\chi}_{f}}$ & $\vartheta$ & $\Psi_{\vartheta}$ & $\bar{\chi}_{f}=\Phi_{\bar{\chi}_{f}}$ & $\Phi_{\vartheta}$ & $\bar{\chi}$ \\
\hline 1 & 2 & 2.236 & 3 & 2.5 & 2.764 & 3 \\
2 & 4 & 4.472 & 5 & 5 & 5.528 & 6 \\
15 & 30 & 33.54 & 34 & 37.5 & 41.46 & 45 \\
100 & 200 & 223.6 & 224 & 250 & 276.4 & 300
\end{tabular}

where $\Psi$ and $\Phi$ are the monotone nonincreasing operators

$$
\begin{aligned}
\Psi: \quad\left[\frac{|V|}{\chi}, \bar{\chi}\right] & \rightarrow[\alpha, \bar{\chi}] \\
\beta & \mapsto \Psi_{\beta}(G)=\min _{t \in \mathbb{N}} t \quad \text { s.t. } \beta\left(K_{t} \square \bar{G}\right)=|V(G)| \\
\Phi: \quad[\alpha, \bar{\chi}] & \rightarrow[\alpha, \bar{\chi}] \\
\beta & \mapsto \Phi_{\beta}(G)=|V(G)|-\beta(S(G))
\end{aligned}
$$


The End

Thank You 